\title{
The Posterolaterally Oriented And Laterally Downward Sloping Facet Joint Is A Risk Factor For Degenerative Cervical Spondylolisthesis And Myelopathy
}

Hiromi Kumamaru ${ }^{1}$, Keiichiro lida ${ }^{1,2}$, Takeyuki Saito ${ }^{1}$, Shingo Yoshizaki ${ }^{1}$, Yasuharu Nakashima ${ }^{2}$, Katsumi Harimaya ${ }^{1}$

1Department of Orthopaedic Surgery, Kyushu University Beppu Hospital, Oita, Japan

2Department of Orthopaedic Surgery, Graduate school of Medical Sciences, Kyushu University, Fukuoka, Japan

Correspondence to: Hiromi Kumamaru, Department of Orthopaedic Surgery

Kyushu University Beppu Hospital, 4546 Tsurumibaru, Beppu, Oita, 874-0838, Japan

$$
\begin{gathered}
+81 \text { 977-27-1600 } \\
+81 \text { 977-27-1605 (fax) } \\
\text { kumamaru.hiromi.849@m.kyushu-u.ac.jp (email) }
\end{gathered}
$$

\section{Conflicts of Interest}

The authors declare no potential interests with respect to the research, authorship, and/or publication of this article.

\section{Authors' contributions}

H.K. designed and interpreted results and wrote and prepared the manuscript. H.K., K.I., T.K., and S.Y. contributed to acquisition of data. Y.N. contributed to the conception of the project. K.H. contributed to the conception of the project and interpretation of results. All authors read and approved the final manuscript.

\section{Funding}

The author(s) disclosed receipt of the following financial support for the research, authorship, and/or publication of this article: Grant-in-Aid for Scientific Research (C), Teijin Pharma.

\section{Ethical approval}

This study was approved by the Kyushu University Institutional Review Board (IRB approval No.29-411).

\section{Informed Consent}

All patients provided informed consent in this study. 


\section{Abstract}

\section{Introduction}

3 Facet joints are anatomical structures that are known to be crucial for determining

4 spinal biomechanical motion; however, the potential relationship between facet

5 orientation and the development of cervical spondylolisthesis remains unclear. Thus,

6 in this study, we aimed to explore the relationship between facet orientation and

7 cervical spondylolisthesis as well as myelopathy.

\section{Methods}

9 Facet orientation in the cervical spine was investigated using computed tomography in

10103 patients with cervical myelopathy, and facet inclination was measured on axial,

11 coronal, and sagittal reconstructed images. Patients were divided into anterolisthesis,

12 retrolisthesis, and no spondylolisthesis groups at each intervertebral level (C2/3-C6/7

13 levels).

\section{Results}

15 Facet joints in the anterolisthesis and retrolisthesis groups tended to slope 16 posterolaterally and downward laterally compared with those in the no

17 spondylolisthesis group at C3/4, C4/5, and C5/6 levels $(P<0.001)$.

\section{Conclusions}

19 The posterolaterally oriented and laterally downward sloping facet at $C 3 / 4$ and $C 4 / 5$

20 levels may be a risk factor for the development of cervical spondylolisthesis as well as

21 symptomatic myelopathy.

23 Keywords: cervical degenerative spondylolisthesis, computed tomography, facet joint, 24 myelopathy 
11 Lumbar DSL is a common disease, while cervical DSL is considered a rare one.

12 However, several recent studies have suggested that cervical DSL is more common

13 than previously thought ${ }^{2}$. The incidence of cervical DSL is reported to be $69 \%$ in elderly

17 Facet joints are crucial anatomical structures in determining the biomechanical

\section{Introduction}

Degenerative spondylolisthesis (DSL) is defined as a pathological condition caused by facet joint arthrosis, disc degeneration, and narrowing of the spinal canal, which could lead to spinal cord compression. Spinal cord compression at the cervical level causes progressive neurological deficit called myelopathy, which then hinders daily living activities. Degenerative cervical spondylolisthesis has been identified to be the primary cause of symptomatic myelopathy, especially in aged people ${ }^{1}$, and the number of degenerative cervical myelopathy (DCM) patients has been increasing with the advancement of an aging society worldwide. patients with DCM who underwent decompression surgery, and the authors concluded that more attention should be paid to cervical $\mathrm{DSL}^{3}$. characteristics of the spine by guiding and restricting spinal motion ${ }^{4}$. Since their orientation is reported to be correlated with spinal movements and clinical conditions ${ }^{5}$, their pathological changes could be associated with abnormal movement of the spinal segment, resulting in DSL. Facet joint orientation at the lumbar spine has been extensively studied, and many studies have indicated that sagittal orientation or facet tropism of the lumbar facet joints is a risk factor for lumbar $\mathrm{DSL}^{6,7}$. However, at the cervical spine level, the relationship between facet orientation and DSL has not been 
1 examined to a great extent.

3 Thus, in this study, we, for the first time, addressed facet joint orientation on sagittal,

4 axial, and coronal planes in patients with DCM using three-dimensional (3D) computed

5 tomography (CT) data to explore the relationship between facet joint orientation and

6 cervical DSL.

8 Material and Methods

\section{Subjects}

10 This study included 103 patients (60 men and 43 women; average age at the time of

11 surgery: 72.5 years [range, 40-94 years]) who underwent expansive open-door

12 laminoplasty for DCM from January 2014 to December 2018 at our institution. Patients

13 with ossification of the posterior longitudinal ligament or the ligamentum flavum,

14 cervical trauma, systemic inflammatory disease, infection, or tumor were excluded

15 from this study. Of the young adults (control), 22 patients who underwent anterior or

16 posterior cervical surgery for cervical disc herniation were included for analysis. There

17 were 17 men and 5 women, and their average age was 43.2 years (range 29-48 years).

18 These patients did not have spondylolisthesis.

20 This study was approved by International Review Board of the authors' affiliated

21 institution.

\section{Radiographic assessment}

24 Before the surgery, standard plain radiographs of the cervical spine including 
1 anteroposterior and lateral radiographs were obtained with the standard tube-to-film

2 distance $(1.8 \mathrm{~m})$ for all patients. Flexion-extension views were obtained from all

3 patients. On lateral radiographs (standing neutral/flexion/extension lateral

4 radiographs), horizontal displacement of one vertebral body relative to the one below

5 it was measured by two independent observers, and the mean values $(\mathrm{mm})$ were then

6 calculated.

8 Anterior slippage was measured by measuring the distance from the posterior-inferior 9 corner of the vertebra above the posterior tangent line along the posterior border of the

10 vertebra below. Posterior slippage was measured in the same way from the posterior-

11 superior corner of the vertebra below the posterior tangent line along the posterior

12 border of the vertebra above. The criterion for static spondylolisthesis is considered to

13 be a slip of $>2 \mathrm{~mm}$ from the adjacent vertebrae on plain radiographs in a neutral

14 position ${ }^{8}$. Using the same method, the slippage in the flexion and extension views was

15 calculated to assess segmental instability, and slippage $>3.5 \mathrm{~mm}$ was considered as

16 dynamic spondylolisthesis according to the criteria by White et al. ${ }^{9}$ and others ${ }^{8,10-12}$. In

17 this study, spondylolisthesis was defined by static and dynamic spondylolisthesis, and 18 the patients were divided into three groups at each intervertebral level according to 19 spondylolisthesis as follows: anterolisthesis, retrolisthesis, and no spondylolisthesis 20 groups.

\section{CT assessment}

23 CT was used to evaluate facet joint orientation in all patients. Using 3D CT imaging 24 software (Aquarius Software, TeraRecon, Inc., San Mateo, CA), the cervical spine was 
reconstructed in each patient.

3 Axial images were obtained from the plane parallel to the superior end plate of the

4 vertebral body. Coronal images were obtained from the plane perpendicular to both

5 axial and sagittal planes. Sagittal images were then obtained from the plane bisecting

6 the vertebral body. On the axial plane, the angles between the tangential line to the

7 posterior vertebral wall and the line connecting the medial and lateral edges of the

8 articular surfaces of the facet joints were measured bilaterally ${ }^{13}$. The mean value of

9 right and left facet angles was calculated for each segment and defined as the axial

10 facet joint inclination (AFI). Posteromedial orientation was defined as $A F I>0$, while

11 posterolateral orientation was defined as AFI < 0 (Fig. 1).

13 On the coronal plane, the angle between the tangential line to the inferior end plate of

14 the vertebral body and the line connecting the superior and inferior edges of the

15 superior articular surfaces was measured bilaterally, and mean values were defined

16 as the coronal facet joint inclination (CFI). When CFI was $>0$, the facet sloped upward

17 laterally, and when it was $<0$, the facet sloped downward laterally (Fig. 1).

19 On the sagittal plane, the angle between the parallel line of the inferior endplate and 20 the line connecting the superior and inferior edges of the superior articular surfaces

21 was measured bilaterally, and mean values were defined as the sagittal facet joint 22 inclination (SFI; Fig. 1). 
1 Magnetic resonance imaging $(\mathrm{MRI})$ was used to determine the location of the

2 neurological compression. All patients were scanned using a 1.5-T MRI system

3 (Siemens MAGNETOM ESSENZA). High signal intensity areas at the C2/3-C6/7

4 levels of the spinal cord were recorded on sagittal and axial views of T2-weighted 5 images (TR/TE 3500-4500/85-110 ms).

6

3

\section{Statistical analysis}

All values are shown as the mean \pm standard deviation. Multiple-group comparisons were made using two-way analysis of variance (ANOVA) with Bonferroni's multiple comparisons test. Fisher's exact test was used for categorical data. Statistical analyses were performed using JMP Pro 13 (SAS Institute, Cary, NC), and a p-value $<0.05$ was considered to be statistically significant.

\section{Results}

\section{Radiographic evaluation for spondylolisthesis}

Cervical DSL (anterolisthesis and retrolisthesis) was recorded in 66 patients (64.1\%). In total, 30 patients had single-level DSL, while others had multiple-level DSL (two levels, 26 patients; three levels, 9 patients; four levels, 1 patient). Of these 113 cervical DSLs, 5 were at $C 2 / 3,37$ were at $C 3 / 4,35$ were at $C 4 / 5,30$ were at $C 5 / 6$, and 6 were at $C 6 / 7$ (Table 1). Anterolisthesis was the most common at the $C 3 / 4$ and $C 4 / 5$ levels, and retrolisthesis was the most common at the C3/4-C5/6 levels (Table 1). The incidence of spondylolisthesis was similar to that shown in a previous report ${ }^{12}$. Ten dynamic anterolisthesis (>3.5 mm anterior motion: $\mathrm{C} 3,5 ; \mathrm{C} 4,4 ; \mathrm{C} 5,1)$ and five dynamic retrolisthesis (>3.5 mm posterior motion: $\mathrm{C} 3,2 ; \mathrm{C} 4,3$ ) were not visualized on plain 
1 neutral radiographs (<2 $\mathrm{mm}$ slippage). The other cervical vertebrae with anterior or

2 posterior segmental instability slipped anteriorly or posteriorly $(>2 \mathrm{~mm})$ in the same

3 direction on neutral position.

4

5 Evaluation of facet orientation in CT multiplanar reconstruction images

6 Three-dimensional CT has been determined as the most powerful tool for facet joint

7 characterization ${ }^{14}$ due to its sensitivity for assessing osseous anatomy. Thus, we

8 measured facet inclinations of the vertebra at $\mathrm{C} 2 / 3-\mathrm{C} 6 / 7$ levels in the axial, coronal,

9 and sagittal planes using CT multiplanar reconstruction images (Fig. 1).

AFI

$12 \mathrm{AFI}$ at each intervertebral level is shown in Table 2. Anterolisthesis and retrolisthesis

13 groups showed significantly smaller AFI compared with the no spondylolisthesis group

14 at the $C 3 / 4$ and $C 4 / 5$ levels (Fig. 2; ANOVA with Bonferroni correction; $P<0.05$ ). The

15 facet joints of the posterior or anterior slipped vertebral bodies tended to slope 16 posterolaterally compared with the non-slipped vertebral bodies from the $C 3 / 4-C 5 / 6$

17 level (Table 2).

19 CFI

20 The CFI at each intervertebral level is shown in Table 3. The anterolisthesis group

21 showed significantly smaller CFI compared with others at the C3/4 level; meanwhile,

22 the retrolisthesis group showed significantly smaller CFI compared with others at the

$23 \mathrm{C} 4 / 5$ level (Fig. 3; ANOVA with Bonferroni correction; $P<0.05$ ). The facet joints of the 
1 posterior or anterior slipped vertebral bodies tended to slope downward laterally

2 compared with non-slipped vertebral bodies at the $C 3 / 4$ and $C 4 / 5$ levels (Table 3 ).

4 SFI

5 The SFI at each intervertebral level is shown in Table 4. All facet joints sloped

6 posteroventrally in this plane, and SFI was noted to be significantly larger in the

7 retrolisthesis group than in the other groups at the C3/4 and C4/5 levels (Table 4 and $8 \quad$ Fig. 4).

10 We compared the proportion of both AFI negative and CFI negative facets at each

11 level among the three groups. Interestingly, a significantly higher proportion of facets

12 in the retro- and anterolisthesis groups showed negative AFI and CFI compared with

13 those in the no spondylolisthesis group at the $\mathrm{C} 3 / 4, \mathrm{C} 4 / 5$, and $\mathrm{C} 5 / 6$ levels (Table 5;

14 Fisher's exact test, $\mathrm{P}<0.001)$.

The proportion of the negative AFI and CFI facet in young adults without

\section{7 spondylolisthesis}

18 In young adults without spondylolisthesis, the negative AFI and CFI facets were 19 observed in $9.1 \%(2 / 22)$ at $\mathrm{C} 2 / 3,27.3 \%(6 / 22)$ at $\mathrm{C} 3 / 4,45.5 \%(10 / 22)$ at $\mathrm{C} 4 / 5,68.2 \%$ $20(15 / 22)$ at $\mathrm{C} 5 / 6$, and $31.8 \%(7 / 22)$ at $\mathrm{C} 6 / 7$.

22 Facet orientation for intramedullary signal intensity on T2-weighted MRI

23 A high intramedullary signal on T2-weighted MR images represents edema, 24 inflammation, and ischemia in the spinal cord and thus is a diagnostic marker for the 
1 spinal segments of myelopathy ${ }^{15}$. To clarify the association of negative AFI and CFI

2 with the development of symptomatic cervical myelopathy, we investigated axial and

3 coronal facet orientation at the spinal segments with intramedullary spinal changes.

4 Intramedullary spinal change was recorded in 106 spinal segments (C2/3, 2 cords;

$5 \mathrm{C} 3 / 4,34$ cords; C4/5, 35 cords; C5/6, 27 cords; C6/7, 8 cords). In addition, $45.1 \%$ of

6 the anterolisthesis segment and $37.0 \%$ of the retrolisthesis segment showed

7 intramedullary spinal changes compared with $11.6 \%$ of the segment without

8 spondylolisthesis, indicating that not only anterolisthesis but also retrolisthesis causes

9 symptomatic myelopathy.

11 In the spinal segments with intramedullary spinal changes, a significantly higher

12 proportion of facets at $\mathrm{C} 3 / 4$ and $\mathrm{C} 4 / 5$ levels showed negative $\mathrm{AFI}$ and $\mathrm{CFI}$ in the retro-

13 and anterolisthesis groups compared with those of the no spondylolisthesis group (Fig.

145 ; Fisher exact test, $P<0.05)$. In addition, $71.9 \%$ and $68.4 \%$ of spinal segments with

15 intramedullary spinal changes in the retrolisthesis and anterolisthesis groups had 16 negative $\mathrm{AFI}$ and $\mathrm{CFI}$, respectively (retrolisthesis: $\mathrm{C} 2 / 3,0 \%$; $\mathrm{C} 3 / 4,44.4 \%$; $4 / 5$, 17 84.6\%; C5/6, 80.0\%; C6/7, 0\%; anterolisthesis: C2/3, 0\%; C3/4, 66.7\%; C4/5, 62.5\%; $18 \mathrm{C} 5 / 6,100 \%$; $66 / 7,0 \%$ ), although only $27.3 \%$ of spinal segments with intramedullary 19 spinal changes in the no spondylolisthesis group had both negative AFIs and CFIs 20 (C2/3, 0\%; C3/4, 6.3\%; C4/5, 21.4\%; C5/6, 60.0\%; C6/7, 25.0\%). These results

21 indicated that the posterolaterally oriented and laterally downward sloping facet caused 22 the development of cervical myelopathy by the development of retrolisthesis or 23 anterolisthesis (Fig. 6). 
2 DSL occurs due to age-related degenerative changes in the facet joints and discs of

3 the cervical spine. In this present study, we showed that facet orientation in the cervical

4 spine was related to spondylolisthesis in patients with symptomatic DCM requiring

\section{Discussion}

decompression surgery. The posterolaterally oriented facets and laterally downsloping facets, especially at the $\mathrm{C} 3 / 4$ and $\mathrm{C} 4 / 5$ levels, are risk factors for spondylolisthesis development and symptomatic myelopathy (Fig. 6).

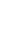

The pathophysiology of DCM is multifactorial, and several causative factors have been proposed for the development of DCM (e.g., degenerative processes, congenital cervical spine stenosis, and spinal alignment) ${ }^{16}$. Tani et al. described the functional importance of degenerative spondylolisthesis in $\mathrm{DCM}^{3}$. Consistent with their study, intramedullary changes were recorded in approximately $40 \%$ of the spondylolisthesis segments in this study. Thus, degenerative cervical spondylolisthesis is considered to cause DCM, and we need to pay more attention to spondylolisthesis in the cervical spine as a causative factor for developing DCM.

It is speculated that the number of cervical DSL patients will increase in the future because spondylolisthesis is secondary to degenerative changes in intervertebral structures, and the population has a longer lifespan than they did in the past. However, the etiology of spondylolisthesis is not well clarified in the cervical spine. A previous study showed that a high T1 slope is a predisposing factor for developing DLS ${ }^{16}$. In addition, the T1-T4 thoracic kyphosis angle was reported to affect DLS ${ }^{17}$. Most previous reports have focused on sagittal parameters, and only a few addressed the 
1 facet orientation on coronal and axial parameters. In this current study, we propose

2 that coronal and axial facet parameters can significantly affect the development of

3 cervical spondylolisthesis in addition to sagittal spinal alignment.

4

5 This present study determined that posterolaterally oriented facets and laterally 6 downward sloping facets were associated with the development of retro- and 7 anterolisthesis at $\mathrm{C} 3 / 4$ and $\mathrm{C} 4 / 5$ levels. This facet orientation may be a predisposing 8 factor for degenerative cervical spondylolisthesis because young people without 9 spondylolisthesis have the posterolaterally oriented and laterally downward sloping 10 facets. These types of facets would be less resistant to vertebral slip due to less 11 protection for intervertebral structures from physiological overload. Anatomically,

12 cervical facet joints allow a greater degree of axial rotation and lateral bending than 13 lumbar facet joints ${ }^{4}$. The posterolaterally oriented facet would then pose less restriction

14 to the antero-posterior translation and could facilitate translation on the sagittal plane

15 in the cervical spine ${ }^{5}$. Axial compressive or tensile loads may not be sufficiently 16 transmitted to the lamina or vertebral body in the posterolaterally oriented facet joint.

17 The laterally downward sloping facets are considered to provide limited bony restriction 18 for axial rotation and lateral bending compared with the laterally upward sloping facet.

19 A combination of these two facet orientations provides less bony restriction to all 20 cervical movement (flexion/extension, axial rotation, and lateral bending), resulting in

21 excessive movement of the intervertebral tissues, such as discs, capsules, and 22 ligaments. Excessive stress on intervertebral soft tissues over a prolonged period 23 could cause the spinal segment to loosen, thus making it unstable. Cervical DSL may 24 then occur without sufficient restriction for the anterior or posterior translation by the 
1 posterolaterally oriented facet ${ }^{5}$.

3 Pal et al. reported that the facet joints gradually or suddenly transition from

4 posteromedial to posterolateral from C2/3 to C7/T1 levels; this was demonstrated by a

5 detailed anatomical analysis of 30 dry macerated normal human male vertebral

6 columns. Their important observation is that once the transition from posteromedial to

7 posterolateral orientation had occurred, reversal of this pattern was never observed in

8 subsequent lower vertebrae ${ }^{18}$. Similar results were reported using a detailed CT

9 analysis $^{19}$. However, this is not the case in this study. Many patients with symptomatic

10 DCM showed a mixed pattern of posteromedially and posterolaterally oriented facets

11 as well as laterally upward and downward sloping facets. Thus, this mixed pattern of

12 cervical facet orientation is considered to be secondary remodeling rather than a pre-

13 existing morphological feature. The remodeling process of facet joint arthritis may alter

14 the morphological features of the facet joints, and the alteration of facet orientation with

15 advancing age seemed to result in cervical DSL.

17 Facet tropism is defined as the angular asymmetry between the paired facet joint 18 orientation, and it is a common phenomenon in the cervical spine ${ }^{5}$. Cervical facet

19 tropism is reportedly associated with cervical spondylolisthesis ${ }^{20}$ as well as cervical 20 disc herniation ${ }^{21,22}$. In this study, no significant relationship was noted between cervical

21 DSL and facet tropism in any planes (data not shown). However, cervical facet tropism 22 may result in asymmetric motion and non-uniform pressure distribution at the 23 intervertebral disc and facet joints, which accelerate disc and facet degeneration ${ }^{5}$. 24 Their degeneration may further increase the mechanical stresses on the intervertebral 
1 disc and facet joints, leading to the instability of the vertebral motion segment, which

2 causes cervical DSL. However, further studies would be needed to clarify the

3 facet tropism and the development of cervical DSL.

4

5 In summary, this study is the first to investigate the relationship between facet

6 orientation and cervical DSL using 3D-CT data. Our results show that the

7 posterolaterally oriented and laterally downward sloping facet may be a predisposing

8 factor for the development of cervical spondylolisthesis and symptomatic myelopathy.

9 However, this study has several limitations. This was a retrospective single-centered

10 study with a relatively small sample size. Young adult controls had cervical disc

11 herniation. We focused on the facet orientation, especially on axial and coronal planes,

12 though many other factors would influence the development of cervical DSL.

13 Nevertheless, strong statistical significance in our study supports our conclusion. In

14 the future, a prospective study is required to evaluate the role of facet orientation in the

15 pathology and etiology of cervical DSL. Further longitudinal clinical studies and

16 biomechanical studies will be necessary to elucidate the causal relationship between

17 facet orientation and the incidence of spondylolisthesis and myelopathy.

18 References

19 1. Young WF. Cervical spondylotic myelopathy: a common cause of spinal cord

20 dysfunction in older persons. Am Fam Physician. 2000;62(5): 1064-70.

21 2. Jiang SD, Jiang LS, Dai LY. Degenerative cervical spondylolisthesis: a

22 systematic review. Int Orthop. 2011;35(6): 869-75. 
1 3. Tani T, Kawasaki M, Taniguchi S, et al. Functional importance of degenerative

2 spondylolisthesis in cervical spondylotic myelopathy in the elderly. Spine (Phila $\mathrm{Pa}$

3 1976). 2003;28(11): 1128-34.

4 4. Jaumard NV, Welch WC, Winkelstein BA. Spinal facet joint biomechanics and

5 mechanotransduction in normal, injury and degenerative conditions. J Biomech Eng.

$6 \quad$ 2011;133(7): 071010.

7 5. Rong X, Liu ZY, Wang BY, et al. The facet orientation of the subaxial cervical

8 spine and the implications for cervical movements and clinical conditions. Spine.

$9 \quad 2017 ; 42(6):$ E320-5.

10 6. Liu X, Zhao X, Long $\mathrm{Y}$, et al. Facet sagittal orientation: possible role in the

11 pathology of degenerative lumbar spinal stenosis. Spine (Phila Pa 1976). 2018;43(14):

$12 \quad 955-8$.

13 7. Liu ZY, Duan YC, Rong X, et al. Variation of facet joint orientation and tropism

14 in lumbar degenerative spondylolisthesis and disc herniation at L4-L5: a systematic

15 review and meta-analysis. Clin Neurol Neurosur. 2017;161: 41-7.

16 8. Kawasaki M, Tani T, Ushida T, et al. Anterolisthesis and retrolisthesis of the

17 cervical spine in cervical spondylotic myelopathy in the elderly. J Orthop Sci. $18 \quad 2007 ; 12(3): 207-13$.

19 9. White 3rd AA, Johnson RM, Panjabi MM, et al. Biomechanical analysis of 20 clinical stability in the cervical spine. Clin Orthop Relat Res. 1975;109: 85-96.

21 10. Dean CL, Gabriel JP, Cassinelli EH, et al. Degenerative spondylolisthesis of the 22 cervical spine: analysis of 58 patients treated with anterior cervical decompression and 23 fusion. Spine J. 2009;9(6): 439-46. 
1 11. Shigematsu $H$, Ueda $Y$, Takeshima $T$, et al. Degenerative spondylolisthesis

2 does not influence surgical results of laminoplasty in elderly cervical spondylotic

3 myelopathy patients. Eur Spine J. 2010;19(5): 720-5.

4 12. Park MS, Moon SH, Lee HM, et al. The natural history of degenerative 5 spondylolisthesis of the cervical spine with 2- to 7-year follow-up. Spine (Phila Pa 6 1976). 2013;38(4): E205-10.

7 13. Cinotti G, Postacchini F, Fassari F, et al. Predisposing factors in degenerative

8 spondylolisthesis. A radiographic and CT study. Int Orthop. 1997;21(5): 337-42.

9 14. Lehman RA, Jr., Helgeson MD, Keeler KA, et al. Comparison of magnetic

10 resonance imaging and computed tomography in predicting facet arthrosis in the

11 cervical spine. Spine (Phila Pa 1976). 2009;34(1): 65-8.

12 15. Nouri A, Martin AR, Mikulis D, et al. Magnetic resonance imaging assessment

13 of degenerative cervical myelopathy: a review of structural changes and measurement

14 techniques. Neurosurg Focus. 2016;40(6): E5.

15 16. Jun $\mathrm{HS}, \mathrm{Kim} \mathrm{JH}, \mathrm{Ahn} \mathrm{JH}$, et al. $\mathrm{T} 1$ slope and degenerative cervical 16 spondylolisthesis. Spine (Phila Pa 1976). 2015;40(4): E220-6.

17 17. Murata K, Endo K, Suzuki $\mathrm{H}$, et al. Spinal sagittal alignment and trapezoidal 18 deformity in patients with degenerative cervical spondylolisthesis. Sci Rep. 2019;9(1): 194992.

20 18. Pal GP, Routal RV, Saggu SK. The orientation of the articular facets of the

21 zygapophyseal joints at the cervical and upper thoracic region. J Anat. 2001;198(4): $22 \quad 431-41$.

23 19. Ebraheim NA, Patil V, Liu JY, et al. Morphometric analyses of the cervical 24 superior facets and implications for facet dislocation. Int Orthop. 2008;32(1): 97-101. 
1 20. Xu C, Lin BQ, Ding ZH, et al. Cervical degenerative spondylolisthesis: analysis of

2 facet orientation and the severity of cervical spondylolisthesis. Spine J. 2016;16(1): 10-

35.

4 21. Huang XC, Ye LQ, Liu X, et al. The relationship between facet tropism and cervical

5 disc herniation. J Anat. 2020;236(5): 916-22.

6 22. Wang $\mathrm{Y}$, Chen $\mathrm{GL}$, Lin JJ, et al. The correlation between facet tropism and

7 intervertebral disc herniation in the subaxial cervical spine. Spine. 2021;46(5): E310-

87.

10 Tables

11 Table 1. Cervical anterolisthesis and retrolisthesis by level

12 Table 2. Axial facet joint inclination (AFI)

13 Table 3. Coronal facet joint inclination (CFI)

14 Table 4. Sagittal facet joint inclination (SFI)

15 Table 5. Proportion of AFI (-) /CFI (-) facets

16 Axial facet joint inclination, AFI; coronal facet joint inclination, CFI

$17 \quad * * * P<0.001$

19 Figure legends

20 Fig. 1. Schema showing the method of measuring the axial, coronal, and sagittal

21 inclination

22 Fig. 2. Axial facet joint inclination (AFI) from $\mathrm{C} 3 / 4$ to $\mathrm{C} 5 / 6$ levels

$23{ }^{*} \mathrm{P}<0.05^{* *} \mathrm{P}<0.01$

24 Fig. 3. Coronal facet joint inclination (CFI) from C3/4 to $\mathrm{C} 5 / 6$ levels 
${ }^{*} P<0.05{ }^{* *} P<0.01$

2 Fig. 4. Sagittal facet joint inclination (SFI) from C3/4 to C5/6 levels

$3 \quad{ }^{* *} \mathrm{P}<0.01$

4 Fig. 5. Proportion of AFI (-) /CFI (-) facets at spinal segments with intramedullary

5 spinal changes

6 Axial facet joint inclination, AFI; coronal facet joint inclination, $\mathrm{CFI}{ }^{*} \mathrm{P}<0.05$

7 Fig. 6. Posterolaterally oriented and laterally downward sloping facets are risk

8 factors for developing spondylolisthesis and symptomatic myelopathy 


\section{Axial facet inclination}

(AFI)

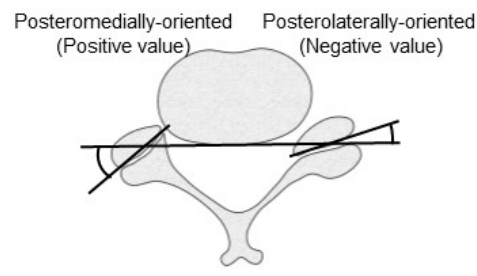

Coronal facet inclination Sagittal facet inclination

(CFI)

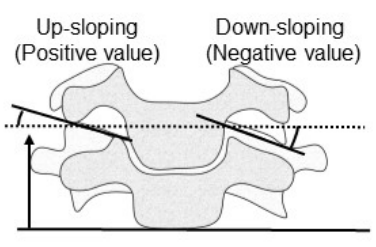

(SFI)

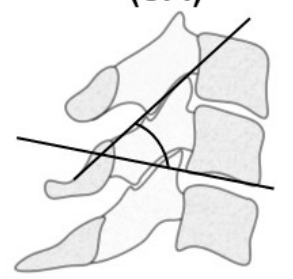

Figure 1. Schema showing the method of measuring the axial, coronal, and sagittal inclination $254 \times 190 \mathrm{~mm}(96 \times 96 \mathrm{DPI})$ 

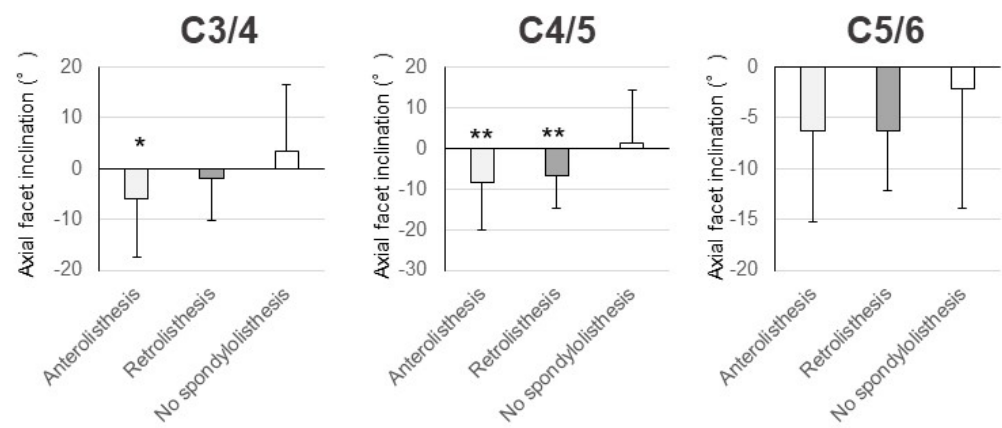

Figure 2. The axial facet joint inclination (AFI) from C3/4 to C5/6 levels $* \mathrm{P}<0.05 * * \mathrm{P}<0.01$ $254 \times 190 \mathrm{~mm}(96 \times 96 \mathrm{DPI})$ 

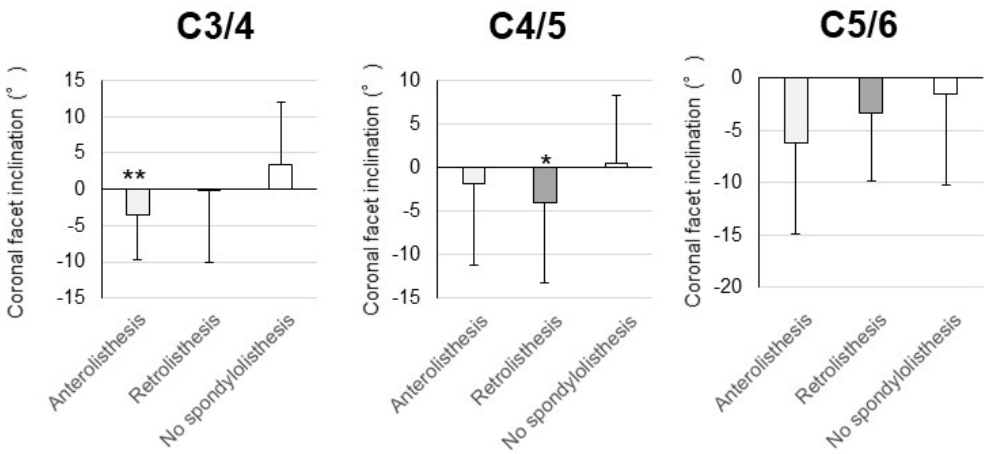

Figure 3. The coronal facet joint inclination (CFI) from C3/4 to C5/6 levels $* \mathrm{P}<0.05 * * \mathrm{P}<0.01$ $254 \times 190 \mathrm{~mm}(96 \times 96 \mathrm{DPI})$ 


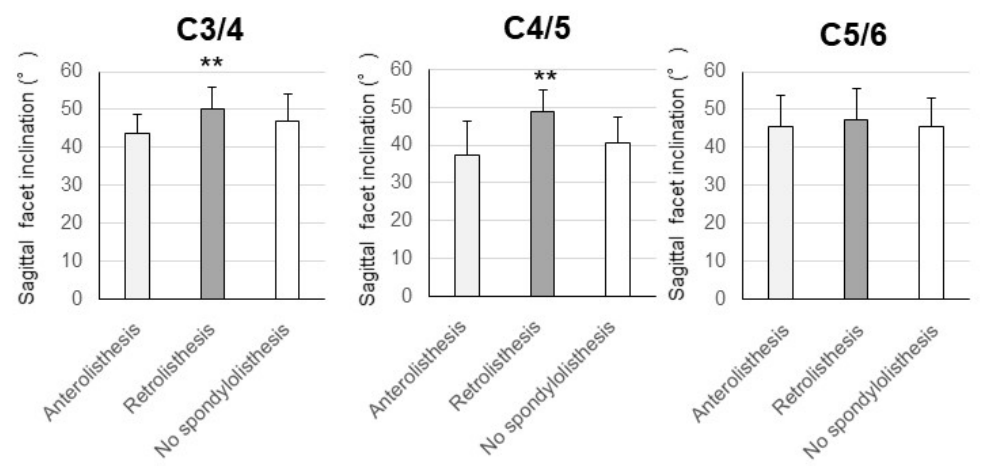

Figure 4. The sagittal facet joint inclination (SFI) from C3/4 to $C 5 / 6$ levels $* * P<0.01$ $254 \times 190 \mathrm{~mm}(96 \times 96 \mathrm{DPI})$ 

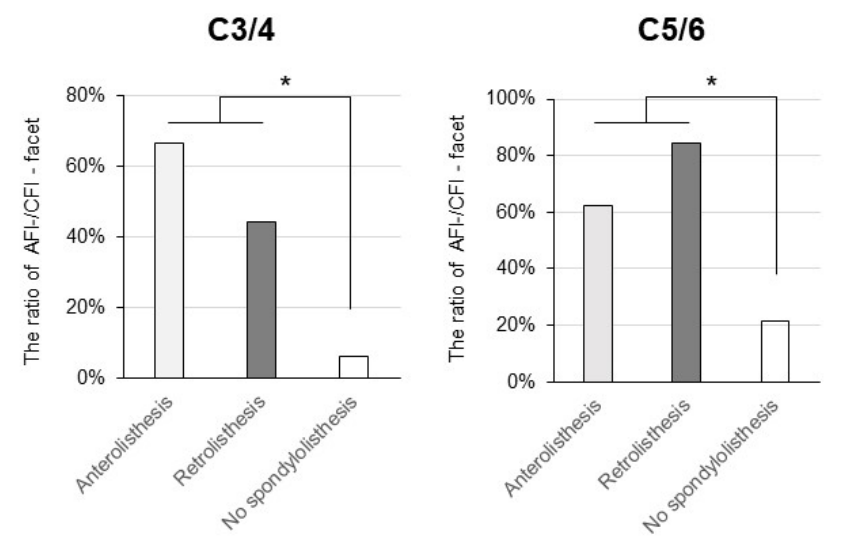

Figure 5. The proportion of AFI (-) /CFI (-) facets at spinal segments with intramedullary spinal changes. Axial facet joint inclination, AFI; coronal facet joint inclination, CFI *P $<0.05$ 
Posterolaterally-oriented facet

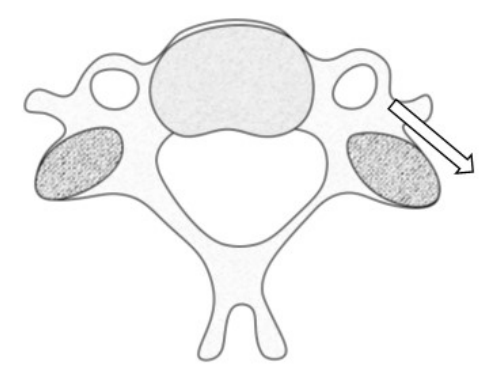

Laterally down-sloping facet

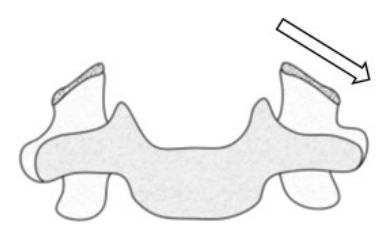

Figure 6. The posterolaterally oriented and laterally downward sloping facet is a risk factor to develop spondylolisthesis and symptomatic myelopathy

$254 \times 190 \mathrm{~mm}(96 \times 96 \mathrm{DPI})$ 
Table 1

\begin{tabular}{|c|c|c|c|c|c|c|}
\hline & C2 & C3 & C4 & C5 & C6 & Total \\
\hline Anterolisthesis & $5(4.9 \%)$ & $12(11.7 \%)$ & $11(10.7 \%)$ & $5(4.9 \%)$ & $1(1.0 \%)$ & $34(6.6 \%)$ \\
\hline Retrolisthesis & 0 & $25(24.3 \%)$ & $24(23.3 \%)$ & $25(24.3 \%)$ & $5(4.9 \%)$ & $79(19.7 \%)$ \\
\hline No spondylolisthesis & 98 & 66 & 68 & 73 & 97 & 402 \\
\hline
\end{tabular}




\section{Table 2}

\begin{tabular}{|c|c|c|c|c|c|c|c|c|c|c|}
\hline & \multicolumn{2}{|c|}{ C2/3 } & \multicolumn{2}{c|}{ C3/4 } & \multicolumn{2}{c|}{ C4/5 } & \multicolumn{2}{c|}{ C5/6 } & \multicolumn{3}{c|}{ C6/7 } \\
\cline { 2 - 11 } & AFI & $\%$ of AFI (-) & AFI & $\%$ of AFI (-) & AFI & $\%$ of AFI (-) & AFI & $\%$ of AFI (-) & AFI & $\%$ of AFI (-) \\
\hline Anterolisthesis & $13.1 \pm 18.2$ & $20 \%$ & $-5.9 \pm 8.8$ & $60 \%$ & $-8.3 \pm 11.6$ & $87 \%$ & $-6.3 \pm 8.9$ & $83 \%$ & 27 & $0 \%$ \\
\hline Retrolisthesis & \multicolumn{2}{|c|}{ N/A } & $-1.9 \pm 11.5$ & $63 \%$ & $-6.6 \pm 8.2$ & $86 \%$ & $-6.3 \pm 5.9$ & $84 \%$ & $-0.4 \pm 6.2$ & $40 \%$ \\
\hline No spondylolisthesis & $21.5 \pm 10.4$ & $3 \%$ & $3.4 \pm 10.4$ & $37 \%$ & $1.4 \pm 13.0$ & $39 \%$ & $-2.1 \pm 11.8$ & $57 \%$ & $2.5 \pm 10.4$ & $35 \%$ \\
\hline
\end{tabular}


Table 3

\begin{tabular}{|c|c|c|c|c|c|c|c|c|c|c|}
\hline \multirow{2}{*}{} & \multicolumn{2}{|c|}{ C2/3 } & \multicolumn{2}{c|}{ C3/4 } & \multicolumn{2}{c|}{ C4/5 } & \multicolumn{2}{c|}{ C5/6 } & \multicolumn{2}{c|}{ C6/7 } \\
\cline { 2 - 12 } & CFI & $\%$ of CFI (-) & CFI & $\%$ of CFI (-) & CFI & $\%$ of CFI (-) & CFI & $\%$ of CFI (-) & CFI & $\%$ of CFI (-) \\
\hline Anterolisthesis & $\begin{array}{c}10.7 \pm \\
15.2\end{array}$ & $20 \%$ & $-3.6 \pm 6.1$ & $67 \%$ & $-1.9 \pm 9.3$ & $73 \%$ & $-6.3 \pm 8.7$ & $83 \%$ & 12.5 & $0 \%$ \\
\hline Retrolisthesis & \multicolumn{2}{|c|}{ N/A } & $-0.2 \pm 9.8$ & $52 \%$ & $-4.0 \pm 9.2$ & $76 \%$ & $-3.3 \pm 6.5$ & $68 \%$ & $4.1 \pm 11.4$ & $40 \%$ \\
\hline No spondylolisthesis & $17.6 \pm 9.4$ & $4 \%$ & $3.5 \pm 8.5$ & $26 \%$ & $0.5 \pm 7.8$ & $38 \%$ & $-1.5 \pm 8.7$ & $60 \%$ & $2.8 \pm 9.0$ & $32 \%$ \\
\hline
\end{tabular}


Table 4

\begin{tabular}{|c|c|c|c|c|c|}
\hline & C2/3 & C3/4 & C4/5 & C5/6 & C6/7 \\
\cline { 2 - 6 } & SFI & SFI & SFI & SFI & SFI \\
\hline Anterolisthesis & $47.6 \pm 6.2$ & $43.7 \pm 5.2$ & $37.5 \pm 8.8$ & $45.4 \pm 8.5$ & 34 \\
\hline Retrolisthesis & N/A & $50.1 \pm 6.0$ & $48.7 \pm 5.8$ & $47.4 \pm 8.2$ & $53.7 \pm 3.1$ \\
\hline No spondylolisthesis & $49.3 \pm 8.3$ & $46.9 \pm 7.2$ & $40.4 \pm 6.8$ & $45.4 \pm 7.7$ & $55.1 \pm 8.1$ \\
\hline
\end{tabular}


Table 5

\begin{tabular}{|c|c|c|c|c|c|}
\hline & $C 2 / 3$ & $C 3 / 4$ & $C 4 / 5$ & $C 5 / 6$ & $C 6 / 7$ \\
\hline Anterolisthesis & $20 \%$ & $60 \%^{* * *}$ & $73 \%^{* * *}$ & $83 \%^{* * *}$ & $0 \%$ \\
\hline Retrolisthesis & N/A & $52 \%^{* * *}$ & $79 \%^{* * *}$ & $68 \%^{* * *}$ & $40 \%$ \\
\hline No spondylolisthesis & $1 \%$ & $21 \%$ & $26 \%$ & $49 \%$ & $1 \%$ \\
\hline
\end{tabular}

